\title{
LEVANTAMENTO DOS FATORES DE RISCO PARA DIABETES MELLITUS TIPO 2 EM UMA INSTITUIÇÃO DE ENSINO SUPERIOR ${ }^{1}$
}

\author{
Maria Carolina Alves Ortiz ${ }^{2}$
}

Maria Lúcia Zanetti

Ortiz MCA, Zanetti ML. Levantamento dos fatores de risco para diabetes mellitus tipo 2 em uma instituição de ensino superior. Rev Latino-am Enfermagem 2001 maio; 9(3):58-63.

0 aumento da expectativa de vida da população, diminuição da atividade física e aumento da ingesta calórica exercem importante papel no aparecimento do diabetes na população. Assim, o objetivo deste estudo é identificar os fatores de risco para o diabetes mellitus tipo 2, em 99 sujeitos de instituição de ensino, em uma cidade do interior paulista. Os resultados permitiram-nos concluir que: 51,5\% dos sujeitos estão com sobrepeso ou obesos; $51,5 \%$ tem antecedentes hereditários, $70 \%$ não realizam atividades físicas regularmente, 68\% sentem-se estressados no trabalho e $6 \%$ apresentam níveis pressóricos alterados. Os resultados indicam a necessidade de programa educativo junto às instituições, visando despertar nos sujeitos a necessidade de buscar estilos de vida saudáveis a fim de prevenir os fatores de risco para o diabetes tipo 2.

UNITERMOS: diabetes mellitus tipo 2, fatores de risco, enfermagem

\section{SURVEY ON RISK FACTORS FOR TYPE-2 DIABETES MELLITUS IN AN UNDERGRADUATION SCHOOL}

The increase in the population's life expectancy, the decrease in people's physical activity and the increase in calorie intake play an important role in the onset of diabetes. Therefore, this study aims at identifying risks factors for type-2 diabetes mellitus in 99 subjects from a teaching institution in a city in the interior of São Paulo State, Brazil. The results enabled the conclusion that $51.5 \%$ of the subjects were overweight; $51.5 \%$ had hereditary antecedents, $70 \%$ did not do physical exercises regularly, $68 \%$ felt stressed at work and $6 \%$ had altered pressure levels. The results showed the need for an education program within the institution aiming at awaking in the subjects the need to look for healthy life styles in order to prevent risks factors for type-2 diabetes.

KEY WORDS: type-2 diabetes mellitus, risks factors, nursing

\section{LEVANTAMIENTO DE LOS FACTORES DE RIESGO PARA LA DIABETES MELLITUS TIPO 2 EN UNA INSTITUCIÓN UNIVERSITARIA}

El aumento de expectativa de vida de la población, la reducción de la actividad física y EL aumento de la ingestión de calorías ejercen un importante papel en el aumento del número de personas con diabetes. De ésta manera, el objetivo del estudio es identificar los factores de riesgo para la diabetes mellitus de tipo 2, en 99 sujetos de una institución de enseñanza, en una ciudad del interior del estado de São Paulo. Los resultados nos permitieron concluir que: 51,5\% de los sujetos están con sus pesos arriba del normal u obesos; 51,5\% tienen antecedentes hereditarios, $70 \%$ no practican ejercicio físico con regularidad, $68 \%$ se sienten muy cansados en el trabajo y $6 \%$ presentan niveles de tensión alternados. Los resultados destacan la necesidad de un programa educativo junto a las instituciones, con el fin de despertar en los sujetos la necesidad de buscar un estilo de vida saludable para prevenir los factores de riesgo de la diabetes del tipo 2.

TÉRMINOS CLAVES: diabetes mellitus tipo 2, actores de riesgo, enfermería

${ }^{1}$ Pesquisa financiada pela FAPESP

${ }^{2}$ Aluna do terceiro ano de graduação em Enfermagem, Bolsista de iniciação científica - FAPESP; ${ }^{3}$ Orientadora do projeto, Professor Doutor. Escola de Enfermagem de Ribeirão Preto da Universidade de São Paulo, Centro Colaborador da OMS para o desenvolvimento da pesquisa em Enfermagem. E-mail: zanetti@eerp.usp.br 
INTRODUÇÃO

diabetes mellitus atualmente é considerado uma das principais doenças crônicas que afetam o homem contemporâneo, acometendo populações de países em todos os estágios de desenvolvimento econômico-social ${ }^{(1-3)}$.

Sua importância nas últimas décadas vem crescendo em decorrência de vários fatores, tais como: maior taxa de urbanização, aumento da expectativa de vida, industrialização, maior consumo de dietas hipercalóricas e ricas em hidratos de carbono de absorção rápida, deslocamento da população para zonas urbanas, mudança de estilos de vida tradicionais para modernos, inatividade física e obesidade, sendo também necessário considerar a maior sobrevida da pessoa diabética.

Pelo impacto social e econômico que tem ocasionado, tanto em termos de produtividade quanto de custos, o diabetes mellitus vem sendo reconhecido, em vários países, como problema de saúde pública com reflexos sociais importantes. Suas manifestações crônicas são ainda, na nossa realidade, causas comuns de hospitalização e absenteísmo no trabalho. Sobressaem, dentre elas, as doenças oculares, renais e vasculares que tem sido apontadas como causas freqüentes de invalidez e incapacitação para o trabalho.

No entanto no Brasil, apesar da importância destes fatos, não dispúnhamos de informaç̃es sobre a prevalência do diabetes mellitus e as possíveis diferenças regionais em sua ocorrência, sendo que somente na última década, foi possível traçar um perfil epidemiológico desta doença na população, confirmando sua importância no cenário da assistência médica hospitalar e ambulatorial, possibilitando incluí-la nas prioridades de saúde e no programa de atenção primária à saúde $e^{(4)}$.

Cabe ainda ressaltar que, em média, metade dos indivíduos brasileiros portadores de diabetes mellitus desconhece sua condição, e que cerca de um quinto dos que a conhecem não realizam qualquer tipo de tratamento. Esta situação pode nos levar a pensar que os indivíduos portadores de diabetes mellitus no Brasil não estão recebendo a atenção de saúde necessária ao seu tratamento e controle $^{(1)}$.

Assim, apesar das dificuldades relacionadas à complexidade que envolve a doença no controle do diabetes mellitus, os programas de controle de saúde devem conter ações individuais e de assistência e ações populacionais de abrangência coletiva, direcionadas à promoção à saúde, a fim de provocar impacto educacional e promover resolutividade.

Atenta à estas questões e considerando que as doenças crônico-degenerativas estão relacionadas ao estilo de vida e aos hábitos sociais e culturais que incidem sobre os indivíduos sobre um longo período, concordamos com as orientações do Ministério da Saúde ${ }^{(5)}$, ao indicar que as intervenções em saúde devam ocorrer antes que os fenômenos patológicos se manifestem. Assim seu controle extrapola as ações assistenciais, necessitando práticas de saúde mais abrangentes, a fim de minimizar o aparecimento dos fatores de risco ou reduzir a oportunidade de exposição das pessoas as esses fatores.

Considerando que a prevenção do diabetes implica na prática de um conjunto de ações para evitar o seu aparecimento ou a sua progressão e que vários fatores de risco para os diabéticos tipo 2 são potencialmente modificáveis, propomos a realização do presente estudo.

\section{JUSTIFICATIVA E OBJETIVOS}

Reconhecendo a importância de que a detecção precoce dos fatores de risco para diabetes mellitus tipo 2 possa minimizar ou reduzir a oportunidade de exposição das pessoas as esses fatores $^{(6)}$, o estudo apresentado tem por objetivos:

- caracterizar os sujeitos segundo as variáveis demográficas e, - identificar os fatores de risco para diabetes tipo 2 em uma instituição de ensino superior.

\section{MATERIAL E MÉTODOS}

0 estudo é de natureza descritiva e seu desenho atende aos pré-requisitos de um estudo de $\operatorname{casos}^{(7-8)}$ e foi realizado na Escola de Enfermagem de Ribeirão Preto da Universidade de São Paulo (EERP- USP).

A população foi constituída por 142 pessoas: 66 docentes e 76 funcionários que trabalham na EERP-USP. Dos 142 funcionários, 05 encontravam-se em licença saúde, 03 aposentados, 02 no exterior e 33 não compareceram ao local estipulado para a coleta no dia pré determinado pelos pesquisadores. Assim, a amostra foi constituída por 99 sujeitos que concordaram em participar do estudo.

As variáveis do estudo foram definidas segundo:

- Função desempenhada: foi considerado docente ou funcionário de acordo com o contrato de trabalho assinado com a instituição de ensino superior;

- Idade: foram elaboradas faixas etárias em intervalos de 10 anos; - Sexo: masculino e feminino; 
- Escolaridade: foi classificada de acordo com a etapa cursada no $1^{\circ}, 2^{\circ}$ e $3^{\circ}$ graus;

- Hereditariedade: foram considerados familiares de $1^{\circ} \mathrm{grau}$ (pais, irmãos) e $2^{\circ}$ grau (avós, tios e primos);

- Índice de massa corporal: para obtenção do IMC foi definido como nível de adiposidade de acordo com a relação peso/altura: onde a relação 20 a 25 está associada ao menor risco de morte prematura ${ }^{(9)}$. A obesidade é categorizada de acordo com três graus: 1 - GRAU I (25 a 29,9), GRAU II (30 a 40) e GRAU III (maior de 40); - Pressão arterial: foi obtida através de aparelho esfigmomanômetro aneróide e estetoscópio Tykos, mediante técnica preconizada ${ }^{(10)}$.

- Proporção da circunferência cintura e quadril: foi utilizada uma fita métrica de plástico MISURA PER SARTI C\&C S.PAULO BRASIL 0 a 150 $\mathrm{cm}$ com intervalos de $0,5 \mathrm{~cm}$, sendo a cintura ou circunferência abdominal definida como a menor circunferência abaixo das costelas e acima do umbigo, e o quadril ou circunferência gluteal como a maior circunferência na extensão posterior das nádegas (AGR), sendo considerada AGR para homens maior que 1,0 e mulheres maior que 0,8 ;

- Estresse em ambiente de trabalho: perguntou-se sobre a presença de estresse no ambiente de trabalho. Foram consideradas de acordo com as respostas dos sujeitos e classificadas em sim e não;

- Nível glicêmico capilar: foi verificada a dosagem do nível glicêmico capilar, por glicosímetro, nos indivíduos que apresentavam-se em jejum por 2 horas ou mais, conforme orientação dada na cartaconvite;

- Atividade física: considerada regularmente quando o sujeito realizava 3 (três) ou mais atividades físicas durante a semana; esporadicamente quando realizava atividade física sem periodicidade e nunca quando referia que não praticava.

Como instrumento de coleta de dados utilizou-se um roteiro sistematizado que foi construído considerando as variáveis do estudo e tomando por base a revisão da literatura ${ }^{(1,3,5,9,11-13)}$ contendo 10 questões fechadas referentes aos dados demográficos e relacionados aos fatores de risco. Referido roteiro foi previamente apreciado em relação à clareza dos itens, facilidade de leitura, compreensão e forma de apresentação, por três enfermeiras.

0 procedimento de coleta de dados obedeceu a seguinte seqüência. Após aquiescência da direção da escola, realizamos um levantamento junto à sessão de Recursos Humanos do número de docentes e funcionários da EERP-USP. De posse da lista com os nomes dos docentes e funcionários, identificamos 142 sujeitos, aos quais foram emitidas cartas contendo os objetivos e a importância do estudo.
Para obtenção do peso e estatura, pressão ar terial e nível glicêmico capilar utilizamos a balança antropométrica Filizola, aparelho esfigmomanômetro aneróide e estetoscópio Tykos, glicosímetro da Advantage, respectivamente, após terem sido submetidos a testes de calibragem.

Os dados foram obtidos através de entrevista a partir de perguntas precisas, pré-formuladas e em ordem pré estabelecida ${ }^{(14)}$, seguida de coleta de dados com utilização de instrumentos previamente calibrados.

Os dados foram coletados no Laboratório 1 da instituição, em dia pré-determinado pelo pesquisador. As entrevistas tiveram duração média de vinte minutos. A coleta de dados foi realizada em março de 1998. As respostas às questões fechadas e os valores obtidos foram registrados no próprio instrumento, concomitantemente à realização dos mesmos.

Aos dados obtidos foram atribuídos códigos específicos para as variáveis demográficas (questões: 1,2,3,4) e as relacionadas aos fatores de risco (questões 5,6,7,8,9,10,11,12). As questões assim codificadas foram transpostas para uma planilha. A estrutura do banco de dados foi confeccionada a partir das planilhas sendo formatado no programa excel. Para a análise dos dados relacionados aos fatores de risco utilizamos os critérios para detectar diabetes mellitus em indivíduos assintomáticos ${ }^{(15)}$.

\section{RESULTADOS E DISCUSSÃO}

Dos 99 docentes e funcionários da instituição de ensino superior investigados, $56,5 \%$ são funcionários e $43,5 \%$ docentes.

Em relação ao sexo, a maioria $(79,8 \%)$ dos sujeitos é do sexo feminino e $20,2 \%$ masculino. Estudos ${ }^{(1,3)}$ mostram que a prevalência de diabetes mellitus é maior no sexo feminino, porém essa diferença não é estatisticamente significante. A incidência e a prevalência do diabetes tipo 2 é 1,4 a 1,8 vez mais freqüente nas mulheres do que nos homens ${ }^{(16)}$. Considerando que a maioria dos sujeitos do estudo é do sexo feminino, programas educativos visando a detecção precoce do diabetes mellitus tipo 2 devam ser incrementados.

Em relação à idade, 83,8\% encontra-se na faixa etária entre 30 e 50 anos (Figura 1). 


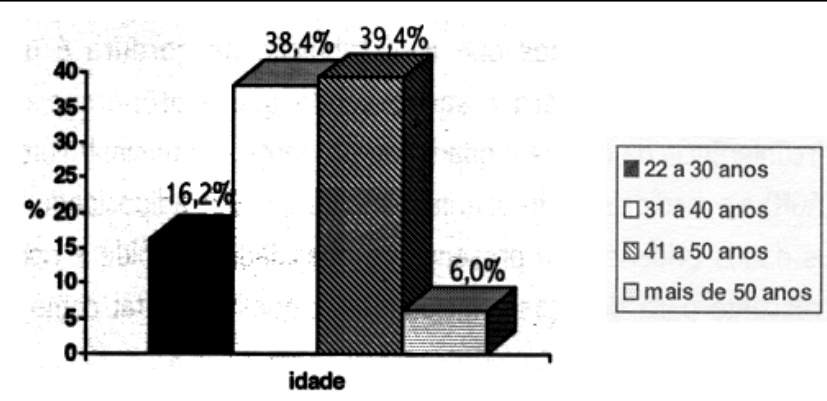

Figura 1 - Distribuição de docentes e funcionários da instituição de ensino superior segundo a idade, Ribeirão Preto-SP, 1998

A incidência e prevalência do diabetes tipo 2 aumenta acentuadamente com o progredir da idade, particularmente, após os quarenta anos ${ }^{(16)}$. Considerando que o diabetes tipo 2 é característico da idade adulta, sendo que, sua incidência é progressivamente maior com o processo de envelhecimento, programas de educação e saúde nas instituições públicas e privadas devem ser promovidos afim de detectar precocemente a doença. Quanto à escolaridade, a maioria, $(61,6 \%)$ têm o terceiro grau completo. Passaremos, em seguida, à caracterização dos sujeitos, segundo as variáveis relacionadas aos fatores de risco.

Em relação a presença de diabetes, a maioria $(72,8 \%)$ refere não ter diabetes, 24,2\% não sabem informar e 3\% são portadores da doença.Quanto ao índice de massa corporal (IMC), $48,5 \%$ estão classificados como normais; $43,4 \%$ sobrepeso e $8,1 \%$ obesos (Figura 2).

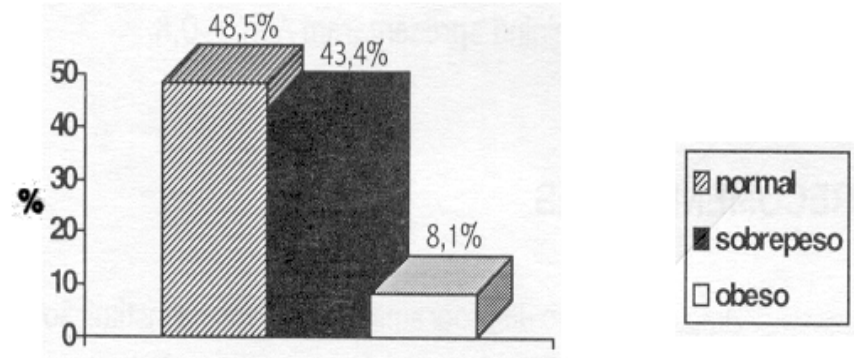

\section{IMC}

Figura 2 - Distribuição de docentes e funcionários da instituição de ensino superior, segundo a classificação de índice de massa corporal, Ribeirão Preto-SP, 1998

Assim, 51,5\% estão acima do índice de massa corporal esperado. Desse modo quando o índice de massa corporal for maior ou igual a $27 \mathrm{Kg} / \mathrm{m}^{2}$, este constitui em um dos critérios para investigar diabetes mellitus em indivíduos assintomáticos ${ }^{(15)}$. Considerando que a obesidade é um importante fator de risco para o diabetes tipo 2 , sendo que, sua freqüência é três vezes maior para o desenvolvimento desta doença, este dado por si só justifica a necessidade de programa de educação para esta população, através dos próprios profissionais da saúde à ela pertencente.
No que se refere à hereditariedade, 50,5\% afirmaram ter familiares com diabetes e $49,5 \%$ que não referem. Dentre os primeiros, 20,2\% relatam ter familiares de primeiro grau portadores da doença. Sabemos que familiares de primeiro grau de diabéticos tipo 2 apresentam de duas a seis vezes mais chance de vir a desenvolver diabetes do que controles sem história familiar ${ }^{(16)}$. Também no diabetes tipo 2 o componente genético é forte, o que é demonstrado pela possibilidade cinco a dez vezes maior de um paciente com história familiar desenvolver a doença em relação à população geral, havendo concordância de $90 \%$ em gêmeos univitelinos $^{(11)}$.

Em relação à atividade física, os dados apontam que $69,7 \%$ dos sujeitos investigados têm dificuldades para realizar atividades físicas regularmente (Figura 3).

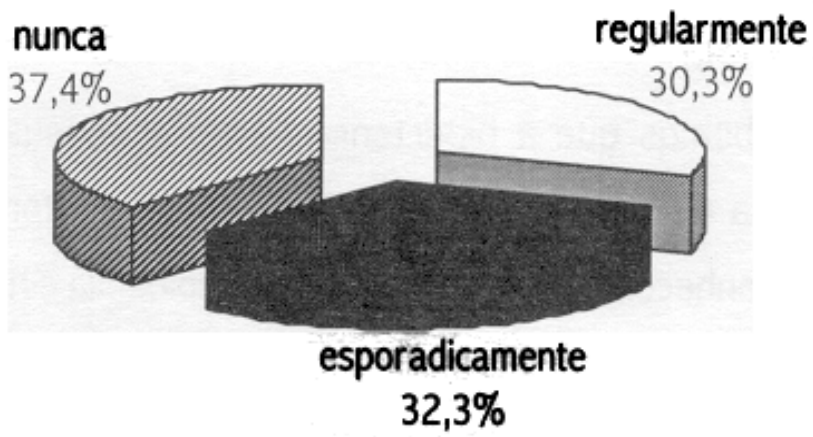

Figura 3 - Distribuição de docentes e funcionários da instituição de ensino superior segundo a atividade física, Ribeirão Preto-SP, 1998

Assim, fatores ambientais como obesidade e sedentarismo têm importante interação com a suscetibilidade genética, colaborando com aumento da resistência à insulina e maior risco de desenvolvimento do diabetes ${ }^{(11)}$. Também o sedentarismo favorece a obesidade, que por si só, é um importante fator de risco para o diabetes tipo $2^{(16)}$. Assim o exercício físico poderá contribuir para reduzir o risco de desenvolver este tipo de diabetes.

Para a maioria $(67,7 \%)$ dos sujeitos estudados 0 ambiente de trabalho constitui um fator de estresse.

0 diabetes mellitus atualmente é considerado uma das principais doenças crônicas que afetam o homem contemporâneo e sua importância vem crescendo em decorrência de vários fatores. Dentre estes destacamos mudanças de estilo de vida tradicional para moderno, inatividade física e obesidade ${ }^{(1-3,17)}$. Nesta direção a ansiedade mental e fisiológica pode diminuir a tolerância à glicose, como também precipitar o diabetes em pessoas cuja tolerância já está em declínio ${ }^{(9)}$. Desse modo, o estresse no ambiente de trabalho pode contribuir para o aparecimento desta doença, exigindo das instituições revisão das diretrizes que norteiam o trabalho.

Em relação ao nível glicêmico capilar $67 \%$ dos sujeitos apresentam níveis menores que $100 \mathrm{mg} / \mathrm{dl}$ e $33 \%$ valores de $100 \mathrm{a}$ 
$199 \mathrm{mg} / \mathrm{dl}$. Segundo os critérios de diagnóstico e classificação do diabetes mellitus ${ }^{(16)}$ para confirmação do diabetes dos sujeitos que apresentam sinais e sintomas e ou condições de risco, os valores de glicemia capilar ao acaso menores que $100 \mathrm{mg} / \mathrm{dl}$ são considerados como diabetes improvável. Nestes casos, recomendase testar a glicemia de jejum a cada dois anos.

Para os valores obtidos entre 100 e $199 \mathrm{mg} / \mathrm{dl}$ recomendase glicemia de jejum. Assim, os dados da presente investigação apontam para a necessidade urgente de acompanhamento dos sujeitos estudados visando a detecção precoce do diabetes.

Em relação aos valores obtidos através da pressão arterial, $6 \%$ dos sujeitos apresentam pressão arterial sistólica (PAS) maior ou igual a $140 \mathrm{mmHg}$ e pressão arterial diastólica (PAD) maior ou igual a $90 \mathrm{mmHg}$.

Sabemos que a hipertensão arterial constitui um dos critérios para detectar diabetes em indivíduos assintomáticos ${ }^{(11)}$. Também reconhecemos que a obesidade, dislipidemia e hipertensão estão associadas ao diabetes mellitus tipo 2 e que o exercício provoca moderada queda da pressão arterial $^{(18)}$.

Isto nos leva a pensar que um programa de atividade física deva ser incorporado junto às atividades dos sujeitos estudados, a fim de prevenir os fatores de risco para o diabetes, incluindo, redução de peso, melhora do estresse e normalização dos níveis pressóricos, visando melhor qualidade de vida.

Em relação aos valores obtidos através da mensuração da proporção da circunferência da cintura e do quadril (AGR) constatamos que $5 \%$ dos homens apresentaram AGR maior que $1 \mathrm{e}$ $46,8 \%$ das mulheres apresentaram AGR maior que 0,8 (Figura 4).

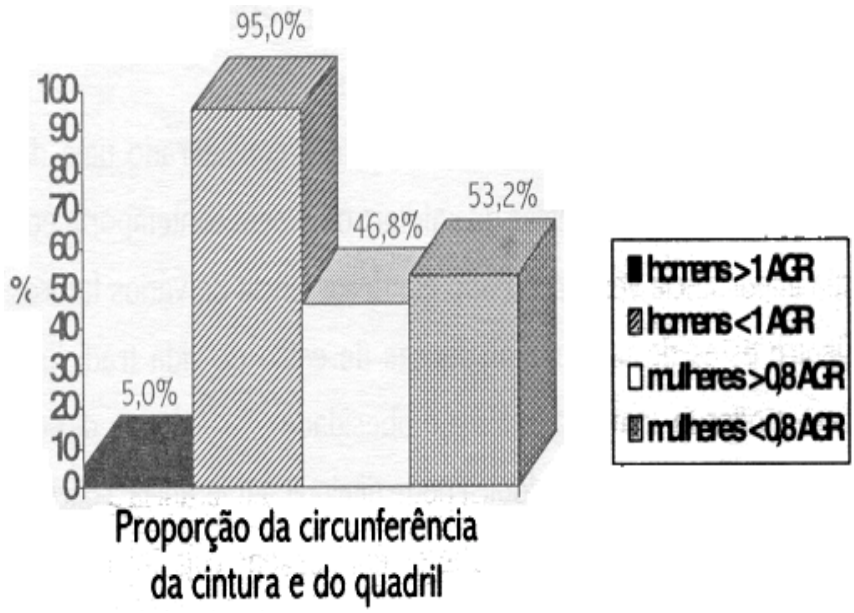

Figura 4 - Distribuição de docentes e funcionários da instituição de ensino superior segundo a proporção da circunferência da cintura e do quadril, Ribeirão Preto-SP, 1998
Reconhecemos que a distribuição de gordura é um indicador de risco para a saúde sendo que a proporção da circunferência da cintura e quadril ou proporção abdominal/glútea (AGR) é a medida mais freqüentemente utilizada de adiposidade ${ }^{(9)}$. Os dados evidenciam a presença de obesidade andróide e risco crescente para doenças relacionadas à obesidade, tal como 0 diabetes mellitus.

\section{CONCLUSÃO}

Apresentamos as conclusões desta investigação de acordo com a seqüência dos objetivos propostos.

\section{Quanto às variáveis demográficas}

Dos sujeitos investigados $56,5 \%$ são funcionários e $43,5 \%$ docentes, 83,8\% encontra-se na faixa etária acima de 30 anos, $79,8 \%$ feminino e $61,6 \%$ tem o $3^{\circ}$ grau completo.

\section{Quanto às variáveis relacionadas aos fatores de risco}

De acordo com o IMC 51,5\% estão com sobrepeso ou obesidade; $51,5 \%$ têm forte componente hereditário; $69,7 \%$ não realizam atividade física regularmente; $67,7 \%$ referem sentir estressados no trabalho; $6 \%$ apresentam níveis pressóricos alterados; $5 \%$ dos sujeitos do sexo masculino apresentaram AGR > 1 e $46,8 \%$ do sexo feminino apresentaram AGR $>0,8$.

\section{RECOMENDAÇÕES}

Implementação de programas educativos na instituição em estudo com vistas à promoção da saúde e prevenção de doenças crônico-degenerativas, em particular, o diabetes mellitus através de:

- Realização de teste de glicemia capilar para os sujeitos que apresentaram nível glicêmico $<100 \mathrm{mg} / \mathrm{dl}$ a cada dois anos e glicemia de jejum para os de níveis >ou igual 100 até $199 \mathrm{mg} / \mathrm{dl}$.

- Implementação de atividade física na instituição, no início ou antes do término da jornada de trabalho, a fim de auxiliar na redução de peso.

- Proporcionar atividades culturais e recreação na instituição visando redução do estresse.

- Oferecer curso anual sobre fatores de risco e sintomas do diabetes mellitus.

- Desenvolvimento de pesquisas no sentido de identificar os fatores que possam favorecer ou interferir para obtenção de estilo de vida saudável. 


\section{REFERÊNCIAS BIBLIOGRÁFICAS}

1. Franco $\sqcup$. Estudo sobre a prevalência do diabetes mellitus na população de 30 a 60 anos de idade no município de São Paulo. [dissertação]. São Paulo (SP): Escola Paulista de Medicina; 1988. 2. Pupo AA, Ursich MJM, Rocha DM. Estratégia do tratamento do diabetes. Rev Assoc Med Bras 1986; 32(11-12):208-12.

3. Malerbi DA. Estudo da prevalência do diabetes mellitus no Brasil. [dissertação]. São Paulo (SP): Faculdade de Medicina/USP; 1991. 4. Ministério da Saúde (BR). Estudo multicêntrico sobre a prevalência do diabetes mellitus no Brasil. Brasília (DF): Ministério da Saúde; 1990.

5. Ministério da Saúde (BR). Doenças crônico degenerativas: evolução e tendências atuais. Brasília (DF): Ministério da Saúde; 1988.

6. ADA: Report of the expert committee on the diagnosis and classification of diabetes mellitus. Diab Care 1998; 21(suppl1):S5S19.

7. Triviños ANS. Introdução à pesquisa em ciências sociais: a pesquisa qualitativa em educação. São Paulo (SP): Atlas; 1992.

8. FACHIN 0. Fundamentos de metodologia. São Paulo (SP): Atlas; 1993.

9. Mahan LK, Arlin MT, tradutores. Alimentos, nutrição e dietoterapia. São Paulo (SP): Roca; 1994.
10. Geddes LA, Whistler SJ. The error in indirect blood pressure measurement with the incorrect size of cuff. Am Heart J 1978; 96:48.

11. Bandeira F, Forti A. Diabetes Mellitus tipo 2. In: Bandeira F et al. Endocrinologia: diagnóstico e tratamento. Rio de Janeiro (RJ): Editora Médica e Científica; 1998. p. 151-61.

12. Zanetti ML. 0 diabetes mellitus tipo 1 em crianças e adolescentes: um desafio para as mães e profissionais da saúde. [dissertação]. Ribeirão Preto (SP): Escola de Enfermagem de Ribeirão Preto/USP; 1996.

13. Vaisman M, Tendrich M. Diabetes mellitus - na prática clínica. Rio de Janeiro (RJ): Editora Cultura Médica; 1994.

14. Richardson RJ, Peres JAS, Correa LM, Peres MHM, Wanderley JCV. Pesquisa social: métodos e tendências. São Paulo (SP): Atlas; 1989. 15. American Diabetes Association. Nutrition recomendations and principles for people with diabetes mellitus. Diabetes Care 1997; 20: S14-7.

16. Ministério da Saúde (BR). Secretaria de Assistência a Saúde, Departamento de Assistência e Promoção à Saúde. Coordenação de Doenças Crônico-Degenerativas. Manual de Diabetes. $2^{\mathrm{a}}$ ed. Brasília (DF): Ministério da Saúde; 1993.

17. Rull JA, Zorrilla E, Jadzinsky MN, Santiago JV. Diabetes Mellitus: complicações crônicas. México: Interamericana/Mc Graw - Hill; 1992. 18. Vivolo MA, Ferreira SRG, Hidal JT. Exercício físico e diabete mellito. Rev Soc Cardiol Estado de São Paulo 1996; 6(1):102-8. 